\title{
BMJ Open Timing of access to secondary healthcare services for diabetes management and lower extremity amputation in people with diabetes: a protocol of a case-control study
}

\author{
Claire M Buckley, ${ }^{1,2}$ Fauzi Ali, ${ }^{3}$ Graham Roberts, ${ }^{3}$ Patricia M Kearney, ${ }^{2}$ \\ Ivan J Perry, ${ }^{2}$ Colin P Bradley ${ }^{1}$
}

To cite: Buckley CM, Ali F, Roberts $\mathrm{G}$, et al. Timing of access to secondary healthcare services for diabetes management and lower extremity amputation in people with diabetes: a protocol of a case-control study. BMJ Open 2013;3: e003871. doi:10.1136/ bmjopen-2013-003871

- Prepublication history and additional material for this paper is available online. To view these files please visit the journal online (http://dx.doi.org/10.1136/ bmjopen-2013-003871).

Received 22 August 2013 Accepted 25 September 2013

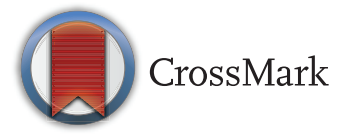

${ }^{1}$ Department of General Practice, University College Cork, Cork, Ireland ${ }^{2}$ Department of Epidemiology \& Public Health, University College Cork, Cork, Ireland ${ }^{3}$ Department of Medicine and Metabolism, Waterford Institute of Technology, Waterford, Ireland

Correspondence to Dr Claire M Buckley; Claire.buckley@ucc.ie

\section{ABSTRACT}

Background: Lower extremity amputation (LEA) is a complication of diabetes and a marker of the quality of diabetes care. Clinical and sociodemographic determinants of LEA in people with diabetes are well known. However, the role of service-related factors has been less well explored. Early referral to secondary healthcare is assumed to prevent the occurrence of LEA. The objective of this study is to investigate a possible association between the timing of patient access to secondary healthcare services for diabetes management, as a key marker of service-related factors, and LEA in patients with diabetes.

Methods/design: This is a case-control study. The source population is people with diabetes. Cases will be people with diabetes who have undergone a first major LEA, identified from the hospital discharge data at each of three regional centres for diabetes care. Controls will be patients with diabetes without LEA admitted to the same centre either electively or as an emergency. Frequency-matching will be applied for gender, type of diabetes, year and centre of LEA. Three controls per case will be selected from the same population as the cases. With a power of $90 \%$ to detect $\mathrm{OR}$ of 0.4 for an association between 'good quality care' and LEA in people with diabetes, 107 cases and 321 controls are required. Services involved in diabetes management are endocrinology, ophthalmology, renal, cardiology, vascular surgery and podiatry; timing of first contact with any of these services is the main exploratory variable. Using unconditional logistic regression, an association between this exposure and the outcome of major LEA in people with diabetes will be explored, while adjusting for confounders.

Ethics and dissemination: Ethical approval was granted by the Clinical Research Ethics Committee of the Cork Teaching Hospitals, Ireland. Results will be presented at conferences and published in peerreviewed journals.
STRENGTHS AND LIMITATIONS OF THIS STUDY

- Data will be based on all patients referred to three regional centres for diabetes covering a well-defined catchment population in the South of Ireland.

- Controls will be selected from the same source population.

- A major predictor of lower extremity amputation in people with diabetes is disease duration. Ideally, controls with a similar duration would be selected. However, this is not possible due to the lack of an appropriate diabetes register or alternative source of this data in the Rol.

\section{Background}

Lower extremity amputation (LEA) is a complication of diabetes and a marker of the quality of diabetes care. LEA rates vary in different communities ${ }^{1}$; the annual incidence of LEA in patients with diabetes in England and the USA was 250 and 450/100 000 people with diabetes, respectively, in 2008. ${ }^{23}$ LEA refers to the complete loss of any part of the lower limb and can be minor or major. A minor LEA is distal to the ankle joint while a major LEA is through or proximal to the ankle joint. ${ }^{4}$ The site of amputation impacts the functional ability and thus the quality of life of the amputee postevent. ${ }^{5}$

Roles of the clinical and sociodemographic risk factors in the development of LEA in people with diabetes have been well described. Long duration of disease, prolonged hyperglycaemia, dyslipidaemia, smoking, neuropathy, peripheral vascular disease and prior ulcers are recognised risk factors for LEA in people with diabetes. ${ }^{6}$ In high-income countries, socioeconomic status 
is associated with the development of diabetes and the subsequent development of LEA. ${ }^{7}$ However, the role of service-related factors has been less well explored.

It has been suggested that the occurrence of LEA is due, in part, to a failed system of healthcare. ${ }^{8}$ The International Diabetes Federation (IDF) estimates that up to $85 \%$ of diabetes-related LEAs could be avoided with timely and appropriate preventive and specialist care. ${ }^{9}$ Worldwide, research suggests that better organised care results in a significant improvement in LEA rates in patients with diabetes. ${ }^{10-12}$

Debate continues on the most appropriate model of care for the management of diabetes. ${ }^{13} 14$ Traditionally, the Irish model for diabetes care was a hospital-led acute illness model. ${ }^{15}$ All patients with type 1 diabetes and the majority of patients with type 2 diabetes were managed in the hospital setting and this has traditionally been considered the best available care. However, not all patients with diabetes can be managed in secondary care and there is a need to consider the optimal timing of referral from primary to secondary care. Timing of access to secondary healthcare and the subsequent occurrence of LEA has not been addressed in previous studies. Thus, the objective of this study is to investigate a possible association between the timing of patient access to secondary healthcare services for diabetes management and the occurrence of LEA, using a casecontrol study design.

\section{METHODS}

\section{Design}

A cohort study is the ideal design for this research question. However, LEAs in patients with diabetes are rare events with long latency periods, so a sufficiently large cohort study powered to detect relevant differences is not feasible. In this scenario, a case-control study is a relevant study design. ${ }^{16}$ OR is a suitable measure when an outcome is rare in the population and controls are selected to represent the same source population that gives rise to the cases.

In this study, the source population is people with diabetes in the Republic of Ireland (RoI). Cases will be people with diabetes who have undergone a first major LEA. Ideally, cases would be identified from a diabetes register, if one existed. ${ }^{17}$ Cases will instead be identified from hospital discharge data from three regional centres for diabetes care with a dedicated vascular surgeon between 2006 and 2012. Exclusion criteria are predefined (box 1).

\section{Box 1 Exclusion criteria for study participants}

Lower extremity amputation secondary to trauma or malignancy Residence outside referral area to three regional centres Age $<45$ years
Controls will be patients with diabetes without LEA admitted to the same centre either electively or as an emergency. Frequency matching will be applied for gender, type of diabetes, year and centre of LEA. Three controls per case will be selected.

\section{Sample size}

Calculation of the sample size was based on the outcome of 'good quality care'. Quality of care was assessed with a patient-completed report card based on 10 process-of-care measures carried out in the last year for diabetes: three or more general practitioner visits for diabetes, glycosylated haemoglobin measurement, blood pressure measurement, urine tested for protein, prescription of aspirin and cholesterol-lowering medication, foot and dilated eye examination, consultation with a dietician and smoking assessment. ${ }^{13}$ Assessment of $\geq 7$ process-of-care measures within the last year was considered 'good quality care'. In the Diabetes Quality of Life Study, Collins et $a l^{13}$ assessed the quality of care in a cross-sectional survey of 2049 Irish people with types 1 and 2 diabetes in 2003. The survey response rate was $71 \%(\mathrm{~N}=1456)$. Overall, $41 \% \quad(\mathrm{~N}=597)$ of respondents had $\geq 7$ process-of-care measures assessed within the last year and thus were considered to have received 'good quality care'. There may have been a response bias in the survey with those completing the survey questionnaire being better attenders at medical services. For the purposes of calculating the sample size, we assumed that all non-responders were poor attenders receiving 'poor quality care'. Thus, the prevalence of the exposure of 'good quality care' was $29 \%$ of people with diabetes.

Previous studies in the UK have shown a reduction in the incidence of major amputations of $71.5 \%$ over 5 years in the South Tees area and $82 \%$ over 11 years in Ipswich following changes to diabetes foot care. ${ }^{10} 12$ This corresponds to an estimated OR of 0.28 and 0.18 , respectively. We consider an estimated OR based on these studies as appropriate, as the UK is our closest neighbour and most similar to ourselves in term of sociodemographics. ${ }^{4}$

With a power of $90 \%$ to detect OR of 0.4 for an association between 'good quality care' and LEA in people with diabetes, 107 cases and 321 controls are required.

\section{Data collection}

All data will be extracted from a combination of hospital discharge data (hospital in-patient enquiry (HIPE) $),{ }^{18}$ administration records and laboratory results in the secondary healthcare setting. Timing of access to secondary healthcare services for diabetes management is the exploratory variable. An expert panel convened consisting of representatives from endocrinology, general practice and public health. Secondary healthcare services deemed by the expert panel to be responsible for the management of diabetes were endocrinology, ophthalmology, renal, cardiology, vascular surgery and podiatry. Data on the timing of first contact with secondary 
healthcare services for diabetes management from any of the included services will be extracted from administration records and collected under three categories: no contact or within 1 year prior to the event (late referral), within 2-3 years prior to the event or within 4-7 years prior to the event (early referral).

It is not feasible that data on all covariates implicated in the occurrence of LEA in people with diabetes would be collected in this study; table 1 outlines the covariates that will be included.

\section{Analysis}

Stata V.12C will be used for statistical analysis. Descriptive analysis will be performed, comparing the distribution of variables among cases and controls using standard parametric and non-parametric tests. A comparison of proportions will be performed using the $\chi^{2}$ statistic for categorical variables and the two-tailed, unpaired Student's t test for continuous variables.

Univariate binary logistic regression analysis will be used to explore relationships between predictor variables and the outcome of LEA in people with diabetes. Multiple logistic regression models will explore associations between exploratory variables and LEA in people with diabetes while adjusting for confounders and allowing for statistical interaction. Care needs to be exercised with logistic regression models to include confounders but not intermediate factors on the causal pathway. The expert panel will discuss each covariate as a potential confounder or intermediate factor (figure 1).

The final model will be assessed for collinearity using the variance inflation factor (VIF). Model fit will be assessed with Hosmer-Lemeshow's goodness-of-fit test. For all analyses, a $p$ value of $\leq 0.05$ will be considered to indicate statistical significance.

As cases and controls will be frequency-matched, unconditional logistic regression with matching variables as covariates will be employed. Conditional logistic regression will also be performed to assess for overmatching. ${ }^{19}$ Results of both analyses will be compared.

\section{DISCUSSION}

A number of case-control studies have been conducted where cases are patients with diabetes who have undergone LEA (see online supplementary appendix 1). ${ }^{6}{ }^{20-29}$ Methods previously employed, especially the selection of cases and controls, were carefully considered. Previously, cases were detected from hospital records as consecutive patients with diabetes undergoing LEA or in the community through the National Drug Re-imbursement Register ${ }^{22} 27$ 28; a case was defined as undergoing any type of non-traumatic LEA. Controls were also recruited from hospital and/or community settings in previous studies. Hospital controls were defined as patients with diabetes undergoing a non-traumatic but medically necessary surgery unrelated to diabetes. ${ }^{27}$ Community controls were selected randomly from all patients with diabetes who had not undergone an LEA at the time of the case operation ${ }^{24}$ or from the electoral register. ${ }^{23}$ The number of controls for each case ranged from 1 to $10 .^{2325}$

This study differs from previous case-control studies in the following two ways:

1. An early minor amputation can prevent a later major amputation $^{11}$, and minor amputations may reflect improved quality of care. The Organisation for Economic Cooperation and Development (OECD) now recommends that only major amputations should be used for quality of diabetes care assessment. ${ }^{30}$ Thus, in this study, cases are defined by an incident major LEA.

2. Exposure to medical care has been previously explored in case-control studies. ${ }^{20}{ }^{23}$ However, to the best of our knowledge, timing of access to secondary healthcare services for diabetes management has not been previously measured and an association between this exposure and major LEA has not been investigated.

A major challenge with case-control studies is the identification and enrolment of suitable control subjects. ${ }^{31}$ In this study, controls will be generated from a hospital base. Hospital controls have several appealing features: convenience, low cost to identify and interview, comparable information quality as cases, motivation to participate and comparable healthcare-seeking behaviour. ${ }^{32}$ The use of hospital and community controls would strengthen the conviction of any associations detected. ${ }^{33}$ To reduce selection bias, the inclusion of hospital and community controls was considered in the design phase of this study. However, recruitment from the community

Table 1 Data collection

\begin{tabular}{lll}
\hline & Variables & Source of Data \\
\hline Main exposure variable & $\begin{array}{l}\text { Timing of first contact with secondary healthcare services } \\
\text { for diabetes management }\end{array}$ & Administration records \\
$\begin{array}{ll}\text { Cardiovascular risk factors } \\
\text { Comorbidities }\end{array}$ & $\begin{array}{l}\text { Smoking, hypertension, dyslipidaemia, obesity, high HbA1c } \\
\text { PVD, history of minor LEA, chronic kidney disease, retinopathy, } \\
\text { ischaemic heart disease }\end{array}$ & HIPE laboratory records \\
$\begin{array}{l}\text { Sociodemographic risk } \\
\text { factors }\end{array}$ & Age, gender, SES, marital status & Administration records \\
\hline $\begin{array}{l}\text { HbA1c, glycosylated haemoglobin HIPE, hospital in-patient enquiry; LEA, lower extremity amputation; PVD, peripheral vascular disease; SES, } \\
\text { socio-economic status. }\end{array}$
\end{tabular}




\section{Confounders}

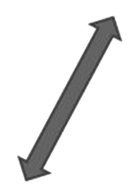

Exposure:

Timing of

Access to

Secondary

Healthcare

Services for

Diabetes

Management

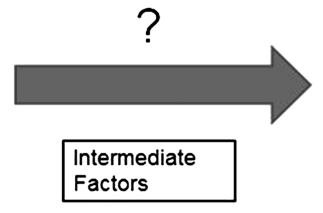

Outcome:

LEA in people

with diabetes

Figure 1 Confounder triangle.

proved impractical due to the lack of a diabetes register in the RoI. ${ }^{17}$

Matching is a technique used to reduce confounding. ${ }^{34}$ Ideally, controls would be frequency-matched for duration of disease to avoid cases having more advanced disease than controls. ${ }^{6}$ To overcome this design issue, we decided that cases will be patients with diabetes admitted as an emergency or electively within the same year to the same hospital as the case.

A person with diabetes selected as a control, who undergoes a major LEA later in the study period, can then be selected as a case and will be included in the study as a control and as a case. A person with diabetes selected as a control will remain in the study population at risk after selection and thus will remain eligible to be selected once again as a control. Including the same person with diabetes at different times does not lead to exposure or confounder information being repeated as this information will change over time. ${ }^{35-37}$

Another potential source of bias in case-control studies is measurement bias. ${ }^{34}$ The reliability of HIPE data has been documented. ${ }^{38}$ Administration data will be collected in the same manner from cases and controls, conforming to the principle of comparable accuracy. ${ }^{39}$

Pilot work suggests that there will be sufficient cases to reach the required sample size at the three included centres. However, if further cases are required, the study area will be extended. If this proves difficult logistically, the number of controls could alternatively be increased.

This study will provide information on the effect of early versus late referral to secondary healthcare services for diabetes management on the outcome of LEA in people with diabetes. While the results will be highly relevant to the Irish healthcare system, the findings will also be generalisable to other countries with hospitalbased, acute illness models of diabetes care. ${ }^{15}$

Contributors CMB conceived and designed the study, gained the funding and wrote the protocol. FA revised the protocol. GR and PMK refined the study design and revised the protocol. IJP and CPB designed the study, gained the funding and revised the protocol. All the authors read and approved the final version of the protocol.
Funding This project is partially funded by the Health Research Board, Ireland (Grant Reference Number: HPF/2009/79) and by the Irish College of General Practitioners (Research and Education Foundation).

\section{Competing interests None.}

Ethics approval Ethical approval was granted by the Clinical Research Ethics Committee of the Cork Teaching Hospitals, Ireland. As data extracted by the researchers were coded with a medical record number around which strict confidentiality safeguards are in place, it was deemed by the Ethics Committee that individual patient consent was not required. All results will be presented at local, national and international conferences and published in peer-reviewed journals. All future publications will follow the Strengthening the Reporting of OBservational studies in Epidemiology (STROBE) criteria. ${ }^{40}$ Patients and policymakers will be informed of results through presentations, reports and updates on the Health Service Executive (HSE) website. It is our hope that these findings will inform service development and redesign.

Provenance and peer review Not commissioned; peer reviewed for ethical and funding approval prior to submission.

Open Access This is an Open Access article distributed in accordance with the Creative Commons Attribution Non Commercial (CC BY-NC 3.0) license, which permits others to distribute, remix, adapt, build upon this work noncommercially, and license their derivative works on different terms, provided the original work is properly cited and the use is non-commercial. See: http:// creativecommons.org/licenses/by-nc/3.0/

\section{REFERENCES}

1. Moxey $P$, Gogalniceanu $P$, Hinchliffe $R$, et al. Lower extremity amputations - a review of global variability in incidence. Diabet Med 2011;28:1144-53.

2. Vamos EP, Bottle A, Edmonds ME, et al. Changes in the incidence of lower extremity amputations in individuals with and without diabetes in England between 2004 and 2008. Diabetes Care 2010;33:2592-7.

3. Margolis DJ, Hoffstad O, Nafash J, et al. Location, location, location: geographic clustering of lower-extremity amputation among medicare beneficiaries with diabetes. Diabetes Care 2011;34:2363-7.

4. Buckley CM, O'Farrell A, Canavan RJ, et al. Trends in the incidence of lower extremity amputations in people with and without diabetes over a five-year period in the Republic of Ireland. PLOS ONE 2012;7: e41492.

5. Peters EJG, Childs MR, Wunderlich RP, et al. Functional Status of Persons with diabetes-related lower-extremity amputations. Diabetes Care 2001;24:1799-804.

6. Gamba MA, Gotlieb SLD, Bergamaschi DP, et al. Lower extremity amputations in diabetic patients: a case-control study. Rev Saúde Pública 2004;38:399-404.

7. Agardh E, Allebeck P, Hallqvist J, et al. Type 2 diabetes incidence and socio-economic position: a systematic review and meta-analysis. Int J Epidemiol 2011;40:804-18.

8. Sussman KE, Reiber G, Albert SF. The diabetic foot problem-a failed system of health care? Diabetes Res Clin Pract 1992;17:1-8.

9. IDF. Position Statement-the Diabetic Foot. http://www.idf.org/ position-statement-diabetic-foot

10. Krishnan S, Nash F, Baker N, et al. Reduction in diabetic amputations over 11 years in a defined U.K. Popul. Diabetes Care 2008;31:99-101.

11. Tseng C-L, Rajan M, Miller DR, et al. Trends in initial lower extremity amputation rates among veterans health administration health care system users from 2000 to 2004. Diabetes Care 2011;34:1157-63.

12. Canavan RJ, Unwin NC, Kelly WF, et al. Diabetes- and nondiabetes-related lower extremity amputation incidence before and after the introduction of better organized diabetes foot care. Diabetes Care 2008;31:459-63.

13. Collins MM, O'Sullivan T, Harkins V, et al. Quality of life and quality of care in patients with diabetes experiencing different models of care. Diabetes Care 2009;32:603-5.

14. Renders CM, Valk GD, Griffin S, et al. Interventions to improve the management of diabetes in primary care, outpatient, and community settings. A systematic review. Diabetes care 2001;24: 1821-33.

15. Brennan C, Harkins V, Perry IJ. Management of diabetes in primary care: a structured-care approach. Eur J Gen Pract 2008;14:117-22.

16. Schulz KF, Grimes DA. Case-control studies: research in reverse. Lancet 2002;359:431-4. 
17. O'Mullane M, McHugh S, Bradley CP. Informing the development of a national diabetes register in Ireland: a literature review of the impact of patient registration on diabetes care. Inform Prim Care 2011;18:157-68.

18. ESRI. Economic and Social Research Institute: Economic and Social Research Institute. http://www.esri.ie/health information/hipe

19. Spitzer WO, Lewis MA, Heinemann LAJ, et al. Third generation oral contraceptives and risk of venous thromboembolic disorders: an international case-control study. BMJ 1996;312:83-8.

20. Del Aguila MA, Reiber GE, Koepsell TD. How does provider and patient awareness of high-risk status for lower- extremity amputation influence foot-care practice? Diabetes Care 1994;17:1050-4.

21. Flores RAR. Risk factors for amputation in diabetic patients: a case-control study. Arch Med Res 1998;29:179.

22. Hamalainen $\mathrm{H}$, Ronnemaa $\mathrm{T}$, Halonen JP, et al. Factors predicting lower extremity amputations in patients with type 1 or type 2 diabetes mellitus: A population-based 7-year follow-up study. J Inter Med 1999;246:97-103.

23. Hennis AJM, Fraser HS, Jonnalagadda R, et al. Explanations for the High Risk of Diabetes-Related Amputation in a Caribbean Population of Black African Descent and Potential for Prevention. Diabetes Care 2004;27:2636-41.

24. Leggetter S, Chaturvedi N, Fuller $\mathrm{JH}$, et al. Ethnicity and Risk of Diabetes-Related Lower Extremity Amputation: A Population-Based, Case-Control Study of African Caribbeans and Europeans in the United Kingdom. Arch Intern Med 2002;162:73-8.

25. Markowitz JS, Gutterman EM, Magee G, et al. Risk of amputation in patients with diabetic foot ulcers: a claims-based study. Wound Repair Regen 2006;14:11-17.

26. Mayfield JA, Reiber GE, Nelson RG, et al. A foot risk classification system to predict diabetic amputation in Pima Indians. Diabetes Care 1996;19:704-9.

27. Reiber GE, Pecoraro RE, Koepsell TD. Risk factors for amputation in patients with diabetes mellitus. A case-control study. Ann Intern Med 1992;117:97-105.
28. Selby JV, Zhang D. Risk factors for lower extremity amputation in persons with diabetes. Diabetes Care 1995;18:509-16.

29. Smith SR, Reed JF III, Greenberg G. Early predictors for lower extremity amputation in a diabetic population: results of a case-controlled study. Int J Low Extrem Wounds 2002;1:170-3.

30. Nicolucci A, Greenfield S, Mattke S. Selecting indicators for the quality of diabetes care at the health systems level in OECD countries. Int J Qual Health Care 2006;18(Suppl 1):26-30.

31. Grimes DA, Schulz KF. Compared to what? Finding controls for case-control studies. Lancet 2005;365:1429-33.

32. Wacholder S, Silverman DT, McLaughlin JK, et al. Selection of controls in case-control studies. Il. Types of controls. Am J Epidemiol 1992;135:1029-41.

33. Yusuf S, Hawken S, Ounpuu S, et al. Effect of potentially modifiable risk factors associated with myocardial infarction in 52 countries (the INTERHEART study): case-control study. Lancet 2004;364:937-52.

34. Grimes DA, Schulz KF. Bias and causal associations in observational research. Lancet 2002;359:248-52.

35. Lubin $\mathrm{JH}$, Gail MH. Biased selection of controls for case-control analyses of cohort studies. Biometrics 1984;40:63-75.

36. Robins JM, Gail MH, Lubin JH. More on "Biased Selection of Controls for Case-Control Analyses of Cohort Studies". Biometrics 1986;42:293-9.

37. Rothman KJ, Greenland S, Lash TL, eds. Modern epidemiology. Wolters Kluwer Health, 2008.

38. Buckley CM, Kearney PM, Ali F, et al. Concordance studies between hospital discharge data and medical records for the recording of lower extremity amputation and diabetes in the Republic of Ireland. BMC Res Notes 2013;6:148.

39. Wacholder S, McLaughlin JK, Silverman DT, et al. Selection of controls in case-control studies. I. Principles. Am J Epidemiol 1992;135:1019-28.

40. Vandenbroucke JP, Elm Ev, Altman DG, et al. Strengthening the reporting of observational studies in epidemiology (STROBE): explanation and elaboration. Ann Intern Med 2007;147:W-163-94. 